\title{
Nora's Lesion in a Child: A Case of Complete Spontaneous Regression
}

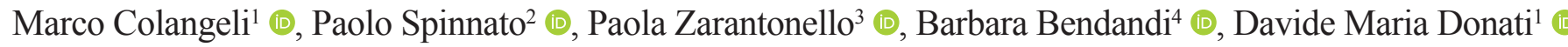

\author{
${ }^{1}$ Department of Musculo-Skeletal Oncology, IRCCS - Istituto Ortopedico Rizzoli, Bologna, Italy \\ ${ }^{2}$ Department of Diagnostic and Interventional Radiology, IRCCS - Istituto Ortopedico Rizzoli, Bologna, Italy \\ ${ }^{3}$ Department of Pediatric Orthopedics and Traumatology, IRCCS - Istituto Ortopedico Rizzoli, Bologna, Italy \\ ${ }^{4}$ Pediatric Emergency Unit, Medical and Surgical Sciences Department, S.Orsola-Malpighi Hospital, University of Bologna, Bologna, Italy
}

We describe a complete spontaneous regression of the Nora's lesion of the first metatarsal in a 3-year-old child with hereditary sensory and autonomic neuropathy type IV (HSNA IV) with congenital insensitivity to pain and anhidrosis. The patient presented at our clinic with a non-tender mass in the dorsal region of the foot. No history of trauma was reported. The skin above the lesion was slightly warm without local bruising. Routine laboratory examinations were unremarkable, except for an elevated C-reactive protein (CRP) level of $6.13 \mathrm{mg} / \mathrm{dL}$ (normal range, $<0.5 \mathrm{mg} / \mathrm{dL}$ ).

$\mathrm{X}$-ray scan and magnetic resonance imaging of the foot revealed a calcified mass adjacent to the cortical surface of the first metatarsal bone (Figure 1). The imaging findings were not specific; therefore, biopsy was performed to achieve an accurate histological diagnosis. Possible differential diagnoses included bizarre parosteal osteochondromatous proliferation (known

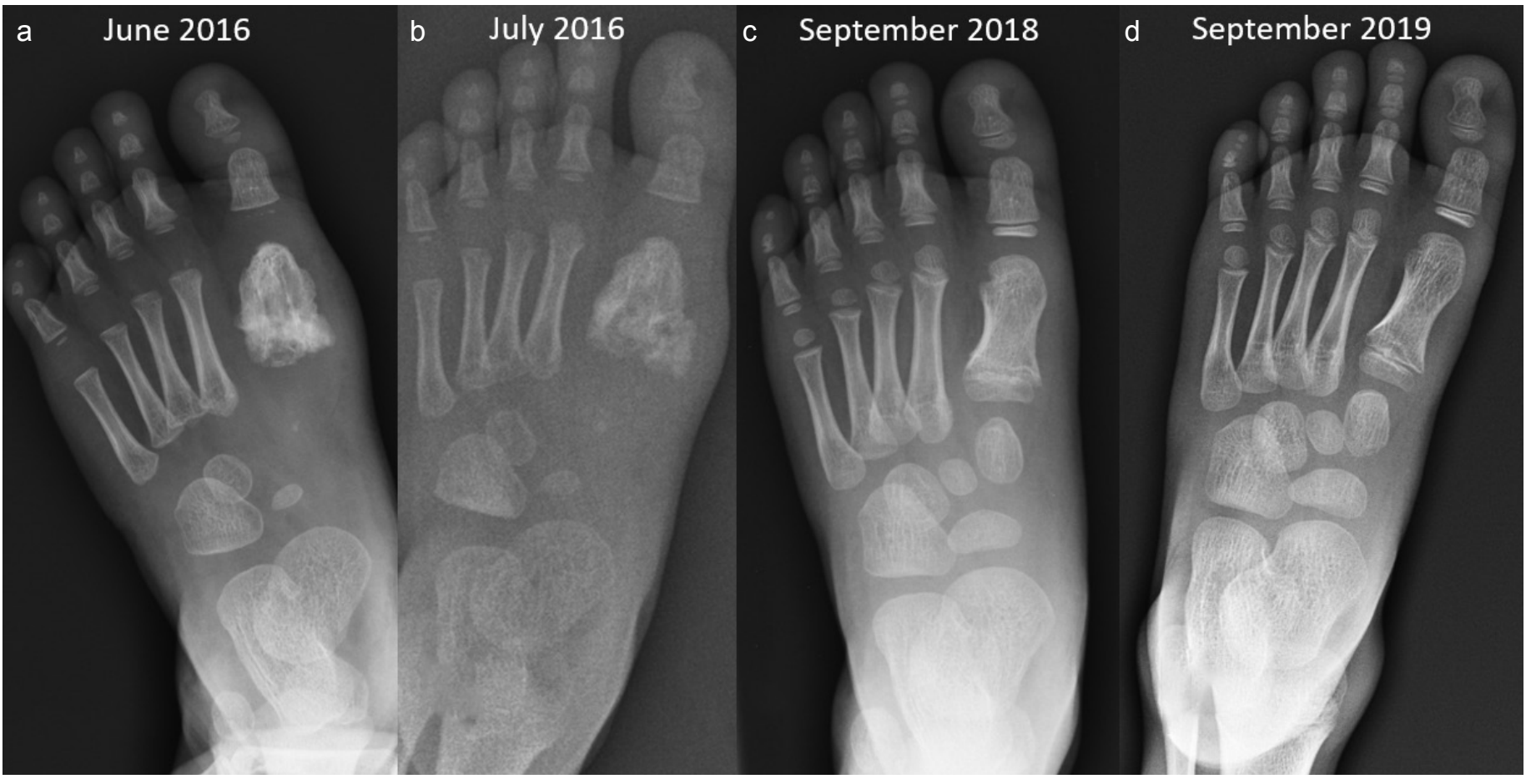

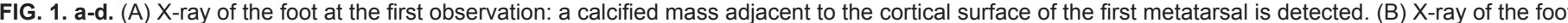

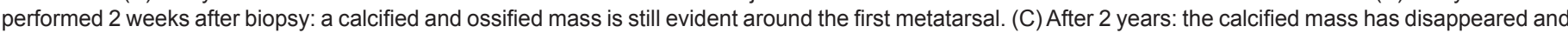

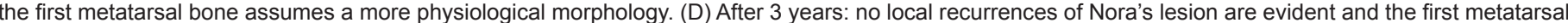
bone shows a completely normal structure

Address for Correspondence: Marco Colangeli, Department of Musculo-Skeletal Oncology, IRCCS - Istituto Ortopedico Rizzoli, Bologna, Italy e-mail: marco.colangeli@ior.it

Received: August 8,2020 Accepted: August 25, 2020 •DOI: 10.4274/balkanmedj.galenos.2020.2020.7.224

Available at www.balkanmedicaljournal.org

ORCID iDs of the authors: M.C. 0000-0003-2772-2807; P.S. 0000-0001-6060-3215; P.Z. 0000-0001-8135-0819; B.B. 0000-0003-1871-9753; D.M.D. 0000-0002-9170-0576.

Cite this article as:

Colangeli M, Spinnato P, Zarantonello P, Bendandi B, Donati DM. Nora's Lesion in a Child: A Case of Complete Spontaneous Regression. Balkan Med J 2021;38:57-8

Copyright@Author(s) - Available online at http://balkanmedicaljournal.org/ 


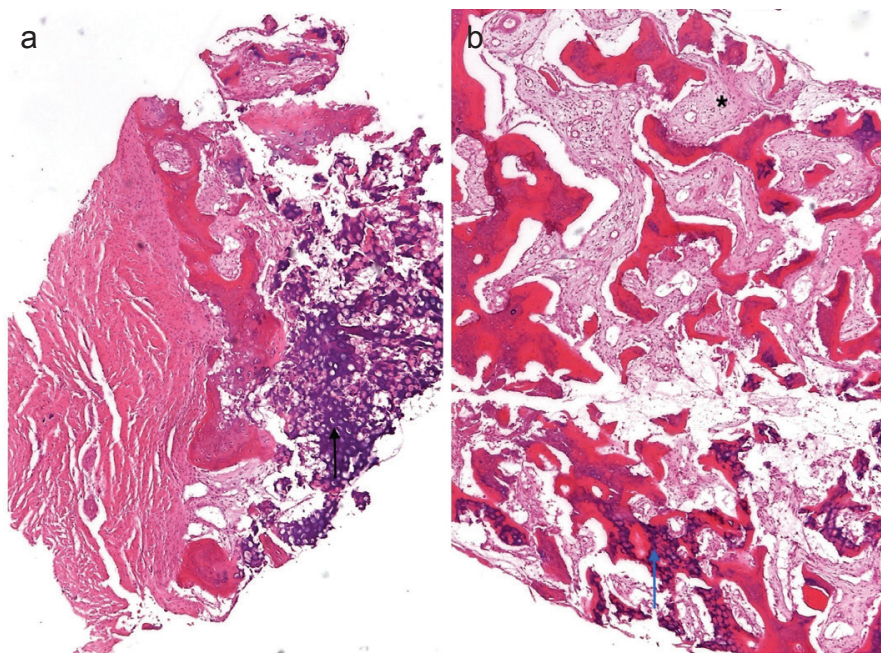

FIG. 2. a, b. Microscopic features of bizarre parosteal osteochondromatous proliferation: (A) Cartilaginous cap with conspicuous areas of basophilic calcifications (black arrow, hematoxylin and eosin staining, x25). (B) Woven bone trabeculae characterized by the presence of so-called "blue bone" (blue arrow) and loose, well-vascularized, and banal spindle-cell stroma present between the bony trabeculae (asterisk, hematoxylin and eosin staining, x50)

as Nora's lesion), periosteal chondroma, osteochondroma, osteomyelitis, myositis ossificans, and parosteal osteosarcoma. Histological examination revealed a diagnosis of Nora's lesion (Figure 2), and intraoperative microbiological examinations were negative for infection. Considering the very young age of the patient, the benign nature of the lesion, and the high recurrence rates $(55 \%)$ reported in the literature (1), we decided to follow a "wait and see" protocol. Surgical treatment would have been considered only in case of an increase in the lesion size. Although an evident source of infection was not found and the blood cultures were negative, an intravenous antibiotic therapy (ceftriaxone, 50 $\mathrm{mg} / \mathrm{kg}$ /day) was started to prevent infection that represents one of the possible complications related to HSNA IV (2). The therapy was continued for 7 days, achieving normalization of the CRP level that remained negative even at the follow-up after 4 and 8 weeks of the antibiotic therapy. We evaluated the patient every 6 months with a clinical and radiological assessment. Surprisingly, the X-ray imaging performed during the follow-up showed a progressive remission of the lesion with complete regression within 2 years (Figure 1).

In this report, we present a pediatric case of a Nora's lesion that showed a progressive, spontaneous, and complete regression. The choice of a "wait and see" approach with clinical and radiological evaluation every 6 months proved to be successful, and a preventive antibiotic therapy avoided any infective complications. Surgical treatment remained an option to be considered only in case of an increase of the lesion size, which did not occur. To the best of our knowledge, this is the first case reported in literature in which Nora's lesion regressed spontaneously (3). This peculiarity makes it original and may support the theory that the Nora's lesion may be a reactive process. In contrast, recent studies have reported some chromosomal anomalies associated with this entity, whose etiopathology remains to be completely understood $(4,5)$.

Patient Consent for Publication: Written informed consent was obtained from the patient.

Acknowledgements: The authors thank Dr. Marco Gambarotti, Dr. Marina Pacheco and Dr. Alberto Righi of the Department of Pathology, IRCCS - Istituto Ortopedico Rizzoli, Bologna (Italy), for providing the image and histological description of the case.

Author Contributions: Concept - M.C., P.S.; Supervision - M.C.; Data Collection and or Processing - P.S.; Analysis and/or Interpretation - D.M.D., M.C.; Literature Search D.M.D., P.Z., B.B.; Writing - M.C., P.S., P.Z., B.B.; Critical Review - M.C., P.S., P.Z., B.B., D.M.D.

Conflict of Interest: The authors have no conflicts of interest to declare.

Funding: The authors declared that this study has received no financial support.

\section{REFERENCES}

1. Joseph J, Ritchie D, MacDuff E, Mahendra A. Bizarre parosteal osteochondromatous proliferation: A locally aggressive benign tumor. Clin Orthop Relat Res 2011;469:2019-27. [Crossref]

2. Axelrod FB, Gold-von Simson G. Hereditary sensory and autonomic neuropathies: Types II, III, and IV. Orphanet J Rare Dis 2007;2:39.doi: 10.1186/1750-1172-2-39. [Crossref]

3. Mahajan S, Chandra R, Mohan Lal Y. "Nora lesion" - Bizarre parosteal osteochondromatous proliferation. J Clin Orthop Trauma 2012;3:119-21. [Crossref]

4. Gruber G, Giessauf C, Leithner A, Zacherl M, Clar H, Bodo K, et al. Bizarre parosteal osteochondromatous proliferation (Nora lesion): A report of 3 cases and a review of the literature. Can J Surg 2008;5:486-9.

5. Michelsen H, Abramovici L, Steiner G, Posner MA. Bizarre parosteal osteochondromatous proliferation (Nora's lesion) in the hand. J Hand Surg Am. 2004;29:520-5. [Crossref] 